\title{
Pulmonary Valvular Endocarditis in the Patient with Pulmonary Stenosis A Case Report with Real-Time 3DTEE\& MDCT-640.
}

\author{
Vu Tuan Nguyen* \\ *PNT Medical University HCMC, Medic HCMC Vietnam, Vietnam \\ *Corresponding author: Vu Tuan Nguyen, PNT Medical University HCMC, Medic HCMC, Vietnam
}

\begin{abstract}
Pulmonary valvular endocarditis is rare disease, the majority of reported cases previously showed isolated PV endocarditis without clear underlying predisposition factors. We report here a 52 ages female patient with endocarditis of pulmonary valve associated with sub valvular pulmonary aneurism, the underlying pulmonary valvular stenosis and RVOT stenosis were confirmed. The 2DTTE, 3D TEE and MDCT-640 were used to make exactly the diagnosis. Patient was under went an un complicated surgical procedure.
\end{abstract}

Keywords: Pulmonary valvular stenosis; TTE: Transthoracic Echocardiography; TEE: Transesophageal Echocardiography; RT3DTEE: Real-time-3DTEE; Endocarditis; Vegetation; MDCT: Multidetector Computed Tomography

\section{Introduction}

Pulmonary valvular stenosis is usually an isolated congenital anomaly and occurs in $7 \%$ to $12 \%$ of patients with congenital heart diseases [1]. and accounts for $80-90 \%$ of native outflow tract obstruction. Severe PS often associated with some degree of sub valvular stenosis resulting from RV hypertrophy. 3DTEE, MRI, Cardiac computed tomography can be used to better define the level of obstruction [2]. Bacterial endocarditis is a rare complication of the disease. Pulmonary stenosis with moderate to severe degree is stratified to intermediate to high risk of endocarditis [3].

\section{Case Report}

A female patient of 52 y.o presented at my hospital by persistent fever for one year, she had had this fever twice daily. Urine infection had been diagnosed and treated by Antibiotics in some hospitals, but the fever had been not interrupted. Finally, she came to MEDIC HCMC. Physical examination detected a 3/6 systolic murmur at the 3rd LICS like VSD. In her past history, no pathological finding has been noted. She was evaluated immediately by a chest X ray that demonstrated a slight prominence of the left second cardiac arch (Figures 1-4). The transthoracic echocardiography revealed a hypertrophic RV with normal TAPSE $=20 \mathrm{~mm}$, normal LV size and function, $E F=71 \%$. Vegetation of $11 \times 5 \mathrm{~mm}$ in size that attached to the pulmonary valve, recorded from parasternal shorts axis view TTE.

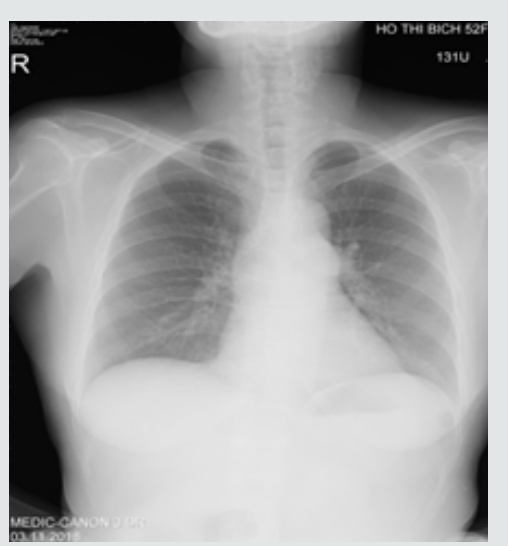

Figure 1: Slighly prominent 2nd left cardiac arch was noted.

The pulmonary cusps were doming and reduced opening degree, a severe pulmonary stenosis with max pressure Gradient $=69 \mathrm{mmhg}$, mean $=42 \mathrm{mmhg}$ was confirmed by continue 
wave Doppler (Figures 5,6). Then Real-time 3DTEE was performed, and we found a mobile vegetation with greater size about $14 \times 7 \mathrm{~mm}$ associated with dilated pulmonary trunk of $35 \mathrm{~mm}$ in diameter and a sub pulmonary valve aneurysm of $25 \mathrm{x} 36 \mathrm{~mm}$ in measuring (Figure 7). CT angiography ( MDCT-640 ) with IV contrast medium Ultravist, slice thickness $=0.5 \mathrm{~mm}$ was indicated and detected a sub pulmonary valve aneurysm of $27 \times 38 \mathrm{~mm}$ and dilated pulmonary trunk: Annulus $=27 \mathrm{~mm}$, Trunk $=40-46 \mathrm{~mm}, \mathrm{RPA}=14 \mathrm{~mm}, \mathrm{LPA}=27 \mathrm{~mm}$. Furthermore, reduced RVOT size revealed: $9.5 \mathrm{~mm}-10.2 \mathrm{~mm}$ in diameter (Figure 8-11). Patient underwent uncomplicated surgical repair: opening RVOT, valvuloplasty and suture the perforated pulmonary cusp (this perforation was not seen prior to operation) with a favorable post-operative progress (Figures 12,13).

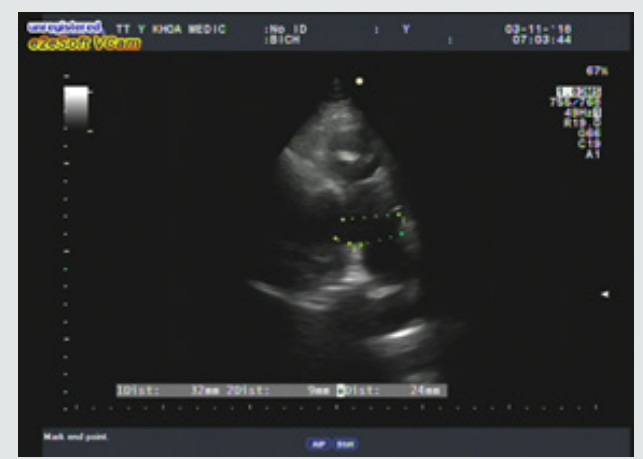

Figure 2: Dilated pulmonary trunk and LPA viewed from 2DTTE parasternal SAX.

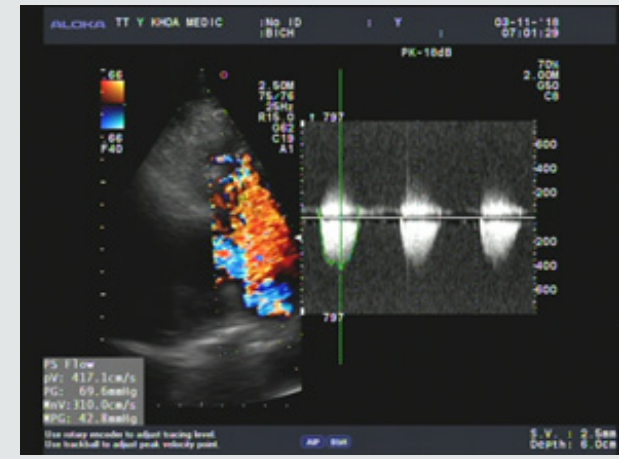

Figure 3: Severe pulmonary stenosis presented by aliasing color Doppler flow and increased transvalvular pressure gradient.

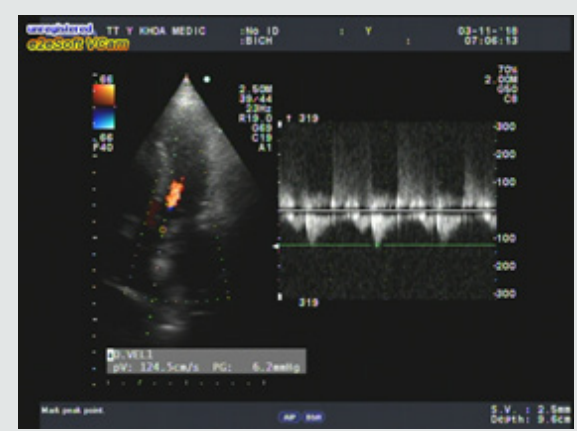

Figure 4: A slight aortic regurgitation recorded from 5C apical view, no aortic valvular vegetation seen.

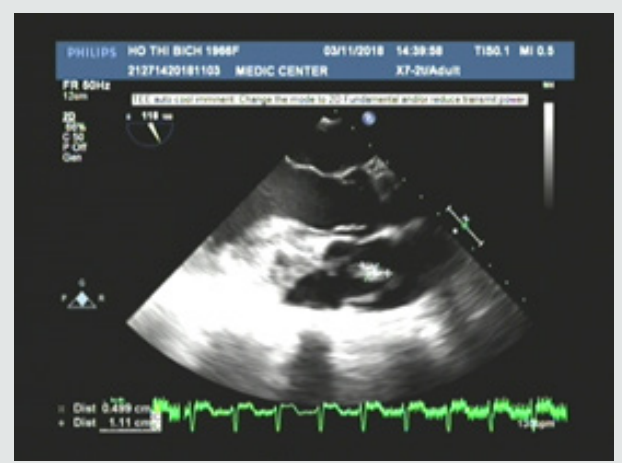

Figure 5: TEE multiplane at $118 \square$ visualized vegetation attached to doming pulmonary valve.

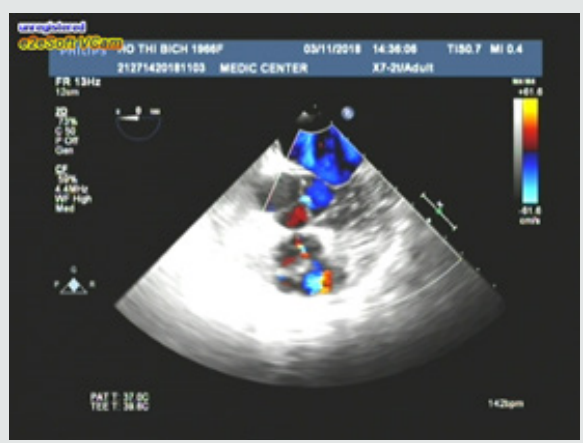

Figure 6: Color Doppler TEE showed PV thickening\& pulmonary regurgitation.

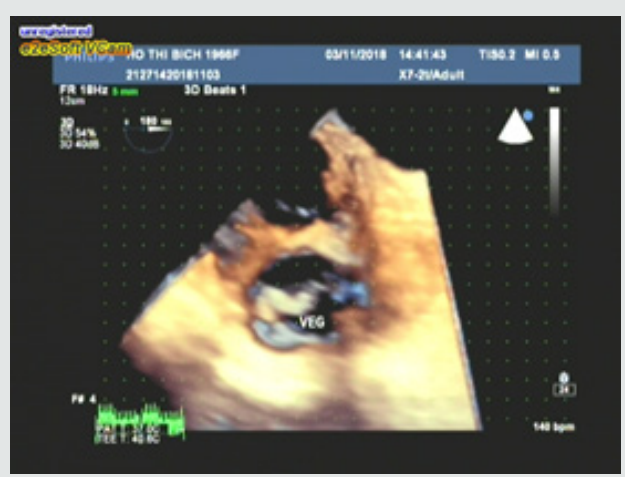

Figure 7: RT-3DTEE demonstrating vegetation better than 2DTEE, particularly shape, size and mobility.

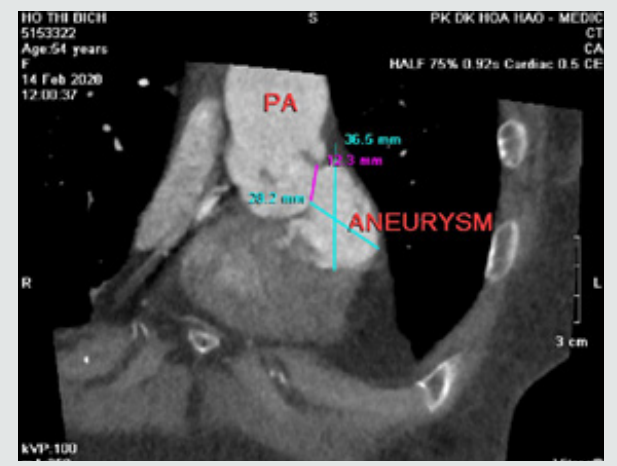

Figure 8: Cardiac MDCT with MPR imaging detected a sub valvular pulmonary aneurysm. 


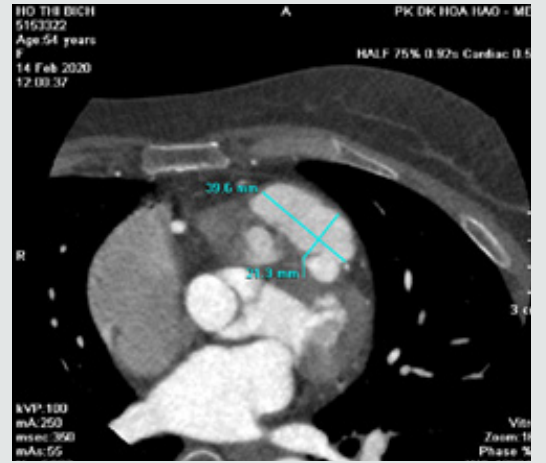

Figure 9: Sub valvular aneurysm with contrast medium inside viewed on axial image.

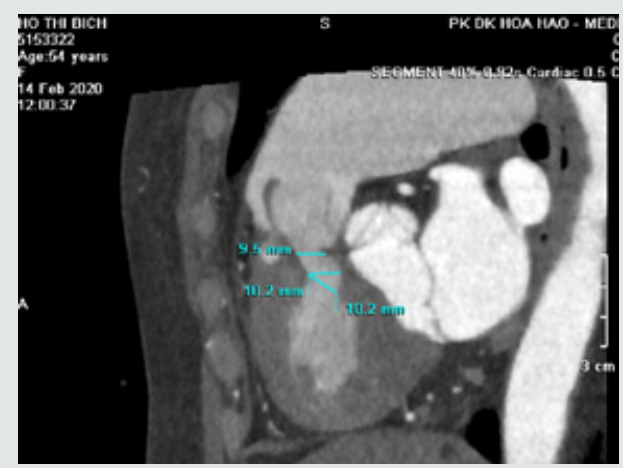

Figure 10: MDCT with sagittal view showed reduced diameter of RVOT.

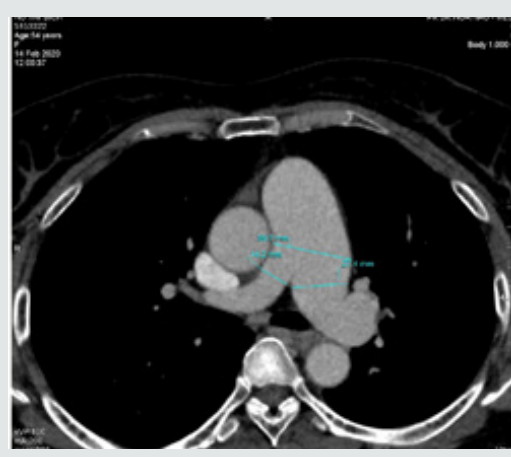

Figure 11: MDCT axial view revealed dilatation of pulmonary trunk and LPA.

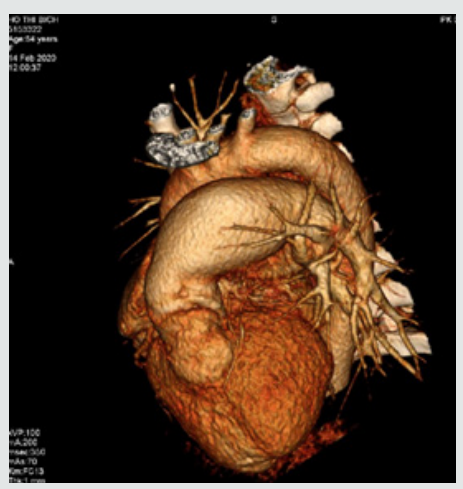

Figure 12: Volume Rendering showed a dilated pulmonary trunk and a sub valvular aneurysm.

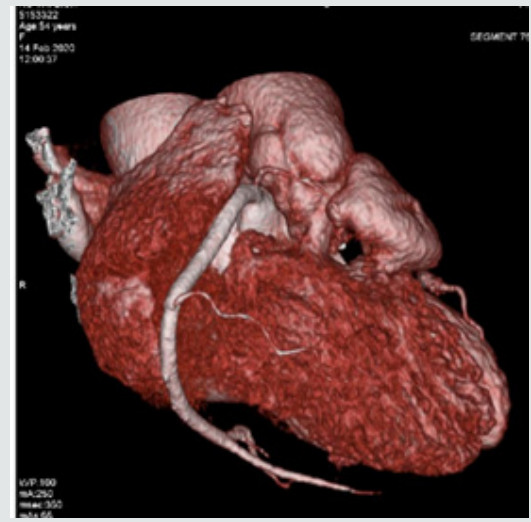

Figure 13: Sub valvular aneurysm viewed from another angle.

\section{Discussion}

Pulmonary valvular infective endocarditis is extremely rare in order to previous publishing. Our patient with pulmonary valvular endocarditis had the underlying predisposition factor including congenital pulmonary valvular stenosis detected on Transthoracic and Transesophageal Echocardiography as domming sign and increased transvalvular pressure gradient. 2DTTE showed a pulmonary valve vegetation that better visualized on the real-time 3DTEE related to its shape, size, mobility. So, 3DTEE provided important information's to make decision of surgical procedure. MDCT-640 in this case was incited for evaluating 2 level of stenosis: RVOT ( decreased size on contrast medium imaging ) and pulmonary valve ( domming sign on systolic imaging ), MDCT also confirmed the diagnosis of sub pulmonary valve aneurysm that possibly resulted from endocarditis complication. Siamak M. Seraj et al. [4]. reported an isolated pulmonary valve endocarditis case, his patient 61 y.o men, presented in condition of acquired pneumonia. Following positive blood culture, Transthoracic Echocardiography confirmed mobile and large pulmonary valve vegetation and a moderate to severe tricuspid regurgitation. The presence of underlying cause was unclear.

Melissa Lyle et al [5]. presented a 63-year-old man that was hospitalized in setting of arthroplasty of chronic knee pain, the past history of patient included CABG at age 59 . His postoperative course was complicated by an Enterococcus faecalis blood stream infection and aortic valve endocarditis, requiring parenteral antibiotics. Patient then presented again for evaluation of recurrent knee pain, a 2/6 early diastolic murmur at the left upper sternal border was detected. TEE revealed a enlarged vegetation attached to a flail pulmonary cusp associated with severe pulmonary regurgitation. The timing of surgery based on the functional capacity and RV function. The decision was made for continued monitoring [6-9].

\section{Conclusion}

Pulmonary valvular endocarditis always are reraly seen and the disease involves to normal as well as abnormal valves like 
congenital valvular stenosis in this case. PV endocarditis could be missed if patients do not present typical clinical features of endocarditis, especially when the predisposition risk factors are absent, or patients abused antibiotics before presented in hospital. The diagnosis of endocarditis mainly based on hemoculture and detecting vegetation. Transthoracic Echocardiography completed by Transesophageal Echocardiography, particularly RT-3DTEE better demonstrating the vegetation. Cardiac MDCT is useful when many levels of obstruction have to been evaluated or endocarditis resulting to severe complications as perforation, aneurysm...

\section{References}

1. Michael A Gatzoulis, Gary D Webb, Piers EF Daubenney (2018) Diagnosis and Management of Adult Congenital Heart Disease, $3^{\text {th }}$ Edition, Elsevier, pp: 460-464.

2. Catherine Otto (2017) The Practice of Clinical Echocardiography , $5^{\text {th }}$ Edition, Elsevier; pp 651-673.
3. Joseph K Perloff (2009) Congenital Heart Disease in Adults, $3^{\text {rd }}$ Edition, Saunsers Elservier pp168-189.

4. Siamak M Seraj, Evan Paul Gill, Simranjit Sekhon (2017) Isolated pulmonary valve endocarditis: truth or myth?. Journal of community hospital internal medicine perspectives 7(5): 329-331.

5. Melissa Lyle, Raul Espinosa (2018) Pulmonary Valve Endocarditis. JACC 71(11): 32863-32868.

6. William F (2010) Armstrong, Thomas Ryan. Feigenbaum's Echocardiography, $7^{\text {th }}$ Edition, William \&Wilkins; pp: 361-384.

7. John R, Haaga, Daniel T, Boll (2017) CT and MRI of the whole body, $6^{\text {th }}$ Edition, Elservier, pp: 2043.

8. Stuart J Hutchison, Naeem Merchant (2015) Principles of cardiac and vascular computed tomography" first Edition, Elservier Saunder 271288.

9. Roberto M, Lang (2012) EAE, ASE Recommendations for Image Acquisition and Display Using Three-Dimensional Echocardiography, JAm Soc Echocardiography 25: 3-46.

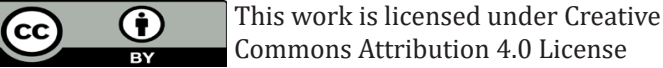

To Submit Your Article Click Here: Submit Article

DOI: $10.32474 /$ ACR.2020.03.000153

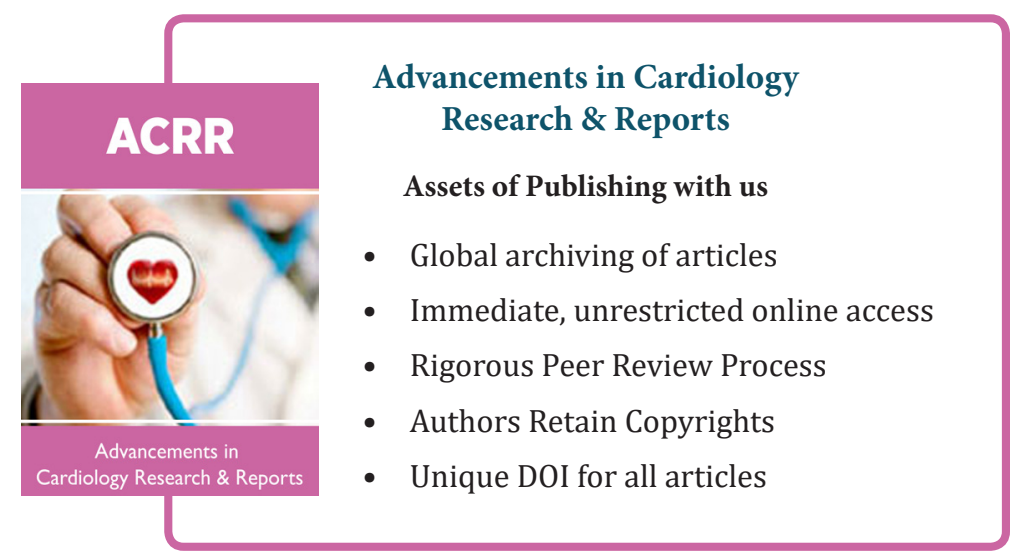

